\title{
A Precise Position and Orientation Algorithm Based on IMM-SINS/GNSS in Aerophotogrammetry System

\author{
Jingxin $\mathrm{Liu}^{\mathrm{a}}$, Yun Bai ${ }^{\mathrm{b}}$, Yang $\mathrm{Ji}^{\mathrm{c}}$
}

\author{
School of Air Force Engineering University Air and Missile Defense College,Xi'an 710051, China

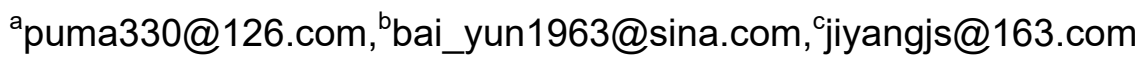

Keywords: Aerophotogrammetry; IMM; SINS/GNSS; Kalman filter; Position and orientation

\begin{abstract}
Aiming at the difficulties of high-precision position and orientation system in aerophotogrammetry system, drift error in traditional Strap-down Inertial Navigation system, noise characteristic mutarions in integrated navigation system, an algorithm based on IMM-SINS/GNSS Kalman filter is proposed. The algorithm takes the advantages of SINS's autonomy and anti-interference characteristics, GNSS's high absolute precision and error-compensating, IMM's multi-model existence and noise adaption characteristic. The error is estimated by using the improved Kalman filter supported by SINS/GNSS integrated navigation system. Simulation results show that the location and attitude error is effectively limited, verify the feasibility of this scheme and the efficiency of the algorithm, showing that this algorithm is valuable in navigation and aerophotogrammetry field.
\end{abstract}

\section{Introduction}

Currently, people are going to have strong need for precise position and orientation. Computing the orientation and position of a carrier, guiding it to our destination, the whole guiding and control process is called navigation. It is necessary to get the space position and orientation of each projective light in aerophotogrammetry system, which is depend on the interior orientation elements in aerial photographic camera. The interior orientation elements is the parameters of aerial photographic camera, and the exterior orientation elements is the six real time parameters of the carrier, including the position and orientation in 3-D space.

With the perfection of GPS/GNSS integrated navigation theory, many researchers applied the integrated navigation system into aerial photogrammetry to accomplish the imaging, at the same time the POS(position and orientation) system is come into being. The POS system combined the SINS(Strap-down Inertial Navigation System) and the GNSS(global navigation satellite system), and offset each other's disadvantages[1,2,3]. It becomes possible to accomplish none or less ground control points in aerial photogrammetry by getting the aerial photographic camera's orientation elements directly via SINS, speeding up the spread and allocation of POS. So it's necessary to carry out an optimizing algorithm to revise the SINS to get precise position and orientation assisting the aerial photogrammetry.

The angular velocity and the accelerated velocity are collected by SINS's gyroscope and accelerometer, the position, velocity and the orientation parameters are calculated via integral. The system can operate independently without outside information all-weather, realize autonomous navigation and anti-interference functions. But it's disadvantages are error accumulation over time, highly dependence. Especially for long time cruise, the accuracy of carrier decrease sharply[4].

GNSS(Global Navigation Satellite System), such as the GPS, Glonass, Galileo and Beidou navigation system. They can provide us high precision position and orientation in 3-D space throughout the world. Their volume is small, mass is low and none error accumulation, but their signal usually disturbed by shelter.

IMM(Interacting Multiple Model) has self-adaptive feature, can solve some problems such as variable-structure and parameter uncertain system state estimation. In response to the question that the mutant of system and measurement noise caused by motivation and environment, the probability of each model can be updated and adjusted swiftly[5,6,7]. On high-mobility state, the 
result of IMM is better than classic Kalman filter[8]. IMM includes several subfilter to accomplish multi-model tracking of the targets.

Considering the characteristics of each navigation system, the IMM, SINS and GNSS are united[9,10,11], proposing a new kind of noise self-adaptive Kalman filter algorithm.

\section{SINS/GNSS integrated navigation model}

2.1 System state equation

1) The model of the accelerometer error

There is only first-order Markov random process of accelerometer drift:

$$
\dot{\nabla}_{a}=-\frac{1}{T_{a}} \nabla_{a}+\omega_{a}
$$

$T_{a}$ is the correlation time, $\omega_{a}$ is the random white noise drift of accelerometer.

2) The model of gyroscope drift error

The gyroscope drift error is composed of random white noise, random first-order Markov process and random constant drift. The error model is as follows:

$$
\varepsilon=\varepsilon_{r}+\varepsilon_{b}+\omega_{g}
$$

Among them, $\varepsilon_{r}$ is the noise of first-order Markov random process, $\dot{\varepsilon}_{r}=-\left(1 / T_{g}\right) \varepsilon_{r}+\omega_{r}, T_{g}$ is the correlation time, $\omega_{r}$ is the first-order Markov random process white noise, $\varepsilon_{b}$ is random constant, and $\dot{\varepsilon}_{b}=0, \omega_{g}$ is the gyroscope random white noise drift.

Let the Geographic coordinate system be the navigation coordinate system, there are 18 variates in this integrated system, including 3 SINS mathematics platform attitude angle error $\phi_{E} 、 \phi_{N} 、 \phi_{N}$, 3 accelerometer error $\delta v_{E} 、 \delta v_{N} 、 \delta v_{U}, 3$ position error $\delta L 、 \delta \lambda 、 \delta h, 3$ gyroscope random constant drift, 3 gyroscope random first-order Markov process drift, 3 accelerometer random Markov process drift. System state equation is as follows:

$$
\dot{X}(t)=F(t) X(t)+G(t) W(t)
$$

The state vectors: $X=\left[\varphi \delta v \delta p \varepsilon_{b} \varepsilon_{r} \nabla\right]^{T}$, random white noise error vectors: $W=\left[\begin{array}{lll}\omega_{g} & \omega_{r} & \omega_{a}\end{array}\right]$.

2.2 Measurement equation

Let the velocity and position error of SINS and GNSS be the observed quantity, the information of SINS is as follows:

$$
\left\{\begin{array} { l } 
{ \lambda _ { I } = \lambda _ { t } + \delta \lambda } \\
{ L _ { I } = L _ { t } + \delta L } \\
{ h _ { I } = h _ { t } + \delta h }
\end{array} \quad \left\{\begin{array}{l}
v_{I N}=v_{N}+\delta v_{N} \\
v_{I E}=v_{E}+\delta v_{E} \\
v_{I U}=v_{U}+\delta v_{U}
\end{array}\right.\right.
$$

The information of GNSS is as follows:

$$
\left\{\begin{array} { c } 
{ \lambda _ { G } = \lambda _ { t } - N _ { E } / R _ { E } \operatorname { c o s } L } \\
{ L _ { G } = L _ { t } - N _ { N } / R _ { M } } \\
{ h _ { G } = h _ { t } - N _ { U } }
\end{array} 、 \left\{\begin{array}{c}
v_{G N}=v_{N}-M_{N} \\
v_{G E}=v_{E}-M_{E} \\
v_{G U}=v_{U}-M_{U}
\end{array}\right.\right.
$$

Among them, $\lambda_{t} 、 L_{t} 、 h_{t}$ are the true value of latitude, longitude and altitude, $v_{N} 、 v_{E} 、 v_{U}$ are the true velocity of the carrier along each axial direction, $N_{E} 、 N_{N} 、 N_{U}$ are the position error of GNSS along each direction, and they are all modified through $R_{E} 、 R_{M}$ and $\cos L$ for matching, their unit is $\mathrm{m} ; M_{E} 、 M_{N} 、 M_{U}$ are the velocity measurement error of GNSS along each direction. The velocity measurement equation is: 


$$
\begin{aligned}
Z_{v}(t) & =\left[\begin{array}{c}
v_{I E}-v_{G E} \\
v_{I N}-v_{G N} \\
v_{I U}-v_{G U}
\end{array}\right]=\left[\begin{array}{l}
\delta v_{E}+M_{E} \\
\delta v_{N}+M_{N} \\
\delta v_{U}+M_{U}
\end{array}\right] \\
& =H_{v}(t) X(t)+V_{v}(t)
\end{aligned}
$$

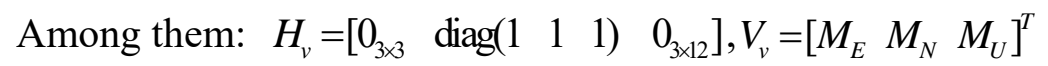

The position measurement equation is:

$$
\begin{aligned}
Z_{p}(t) & =\left[\begin{array}{c}
\left(\lambda_{I}-\lambda_{G}\right) R_{N} \cos L \\
\left(L_{I}-L_{G}\right) R_{M} \\
h_{I}-h_{G}
\end{array}\right]=\left[\begin{array}{c}
R_{N} \cos L \delta \lambda+N_{E} \\
R_{M} \delta L+N_{N} \\
\delta h+N_{U}
\end{array}\right] \\
& =H_{p}(t) X(t)+V_{p}(t)
\end{aligned}
$$

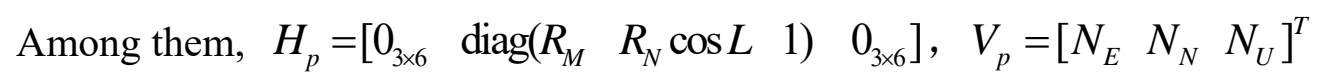

Combine velocity and position measurement equation, SINS/GNSS integrated navigation measurement equation are as follows:

$$
Z(t)=\left[\begin{array}{c}
Z_{p}(t) \\
Z_{v}(t)
\end{array}\right]=\left[\begin{array}{c}
H_{p}(t) \\
H_{v}(t)
\end{array}\right] X(t)+\left[\begin{array}{c}
V_{p}(t) \\
V_{v}(t)
\end{array}\right]
$$

\section{Position and Orientation Algorithm Based on IMM and SINS/GNSS}

\subsection{IMM model}

IMM maps different parameters systems to model set, each filter of each model operates at the same time. Based on the output residual information and prior information corresponding of each filter, follow the principle of hypothesis testing, get the matching probability of each model and the current system model, which is called model probability. The final system state estimation is the weighted fusion of each filter's state[12]. Discrete condition, linear system state equation and measurement equation are as follows:

$$
\begin{gathered}
X(k+1)=F(k+1, k) X(k, m(k))+ \\
G(k) W(k, m(k)), \\
Z(k)=H(k)(X(k), m(k))+V(k, m(k)) .
\end{gathered}
$$

Among them, let the system model set be $M=\left\{m_{1}, m_{2}, \cdots, m_{n}\right\}$, system measurement set be $Z(k)=\{z(1), z(2), \cdots, z(k)\} \cdot m(k)$ is the valid model under the current sampling time $k$, model-to-model transformation obeys the Markov process. $\pi_{i j}$ is the transition probability from $m_{i}(k)$ to $m_{j}(k+1) . \quad \mu_{i}(k)$ is the matching probability for model $m_{i}$ under the sampling time $k$.

The initial Markov transition probability is:

$$
\begin{aligned}
& \pi_{i j}=\operatorname{pr}\left\{m_{j}(k+1) \mid m_{i}(k)\right\}, m_{j}, m_{i} \in M, \\
& \sum_{j=1}^{n} \pi_{i j}=1, i=1, \cdots n .
\end{aligned}
$$

Model prediction probability is:

$$
\mu_{j \mid i}(k-1)=\frac{\pi_{i j} \mu_{i}(k-1)}{\sum_{i=1}^{n} \pi_{i j} \mu_{i}(k-1)}
$$

\subsection{Filter process}

The whole filter process includes 4 step:1) inputs interaction;2) kalman filter;3) model probability update;4) outputs fusion. The flow chart is as follows: 


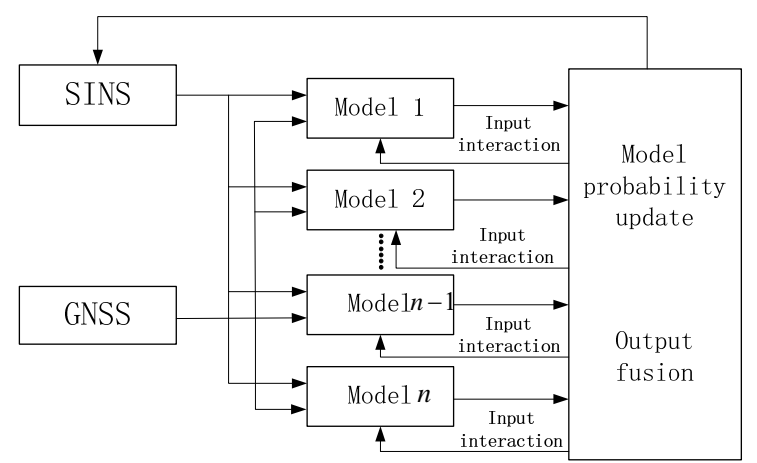

Fig. 1 The filtering flow chart

1) Filter $j$ inputs interaction:

$$
\begin{aligned}
\hat{X}_{o j}(k-1)= & \sum_{i=1}^{n} \hat{X}_{i}(k-1) \mu_{j \mid i}(k-1), \\
P_{o j}(k-1)= & \sum_{i=1}^{n} \mu_{j \mid i}\left\{P_{i}(k-1)+\left[\hat{X}_{i}(k-1)-\hat{X}_{o j}(k-1)\right] \bullet\right. \\
& {\left.\left[\hat{X}_{i}(k-1)-\hat{X}_{o j}(k-1)\right]^{T}\right\} . }
\end{aligned}
$$

Based on all the prior and residual information, each filter's initial value is worked out via the computing of all the model transition probability and state estimation value.

2) Kalman filter:

After the input interaction of filter $j$, the state forecast on next step is:

$$
\hat{X}(k, k-1)=F(k, k-1) X(k-1)
$$

State estimation is:

$$
\begin{aligned}
\hat{X}(k, k-1)= & F(k, k-1) X(k-1)+K(k, m(k)) \\
& (Z(k-1)-H(k) \hat{X}(k, k-1))
\end{aligned}
$$

Filter gain:

$$
\begin{aligned}
K(k, m(k))= & P(k, k-1, m(k)) H^{T}(k)(H(k) P(k, k-1, m(k)) \\
& \left.H^{T}(k)+R(k, m(k))\right)^{-1}
\end{aligned}
$$

New covariance matrix:

$$
\begin{aligned}
S(k)= & H(k) P(k, k-1, m(k)) H^{T}(k)+ \\
& R(k, m(k))
\end{aligned}
$$

Residual error $S(k)=\operatorname{cov}[v(k)] P_{z z}$

Next step prediction mean squared error:

$$
\begin{aligned}
P(k, k-1)= & F(k, k-1) P(k-1) F(k, k-1)^{T} \\
& +G(k-1) Q(K) G(k-1)^{T}
\end{aligned}
$$

Estimation mean squared error:

$$
\begin{aligned}
P(k)= & (I-K(k, m(k)) H(K)) \\
& P(k, k-1, m(k))
\end{aligned}
$$

So far the whole Kalman filter time update and measurement update process are finished, each filter $j$ finish it's job. During the whole process, each filter works in parallel.

3) Model probability updates:

Model probability update use the Bayesian hypothesis testing method, test the residual error of each filter. Due to the Kalman filer theory, the mean value of filter's residual error is 0 and the variance is Gaussian white noise $S(k) \cdot f_{i}(k)$ is the likelihood function for $m_{i}$ being the system matching model at the sampling time $k$. 


$$
\begin{aligned}
f_{i}(k)= & N\left[\varepsilon_{i}(k): 0, S_{i}(k)\right]= \\
& {\left[(2 \pi)^{m}\left|S_{i}\right|\right]^{-0.5} \exp \left(0.5 \varepsilon_{i}^{T} S_{i}^{-1} \varepsilon_{i}\right) }
\end{aligned}
$$

$\varepsilon_{i}$ is the estimation of the residual error and $S_{i}=E\left(\varepsilon_{i} \varepsilon_{i}{ }^{T}\right)=p_{z z}^{i}$ is the expectation of the residual error.

Model probability update is:

$$
\begin{aligned}
u_{j}(k)= & P\left\{m_{i}(k) \mid Z(k)\right\} \\
= & \frac{f_{j}(k) \sum_{i=1}^{n} \pi_{i j} \mu_{i}(k-1)}{\sum_{j=1}^{n} f_{j}(k) \sum_{i=1}^{n} \pi_{i j} \mu_{i}(k-1)}
\end{aligned}
$$

4) Output fusion:

Make the weight fusion of each filter, the result is as follows:

$$
\begin{aligned}
\hat{X}(k)= & \sum_{j=1}^{n} \hat{X}_{j}(k) \mu_{j}(k), \\
P(k)= & \sum_{j=1}^{n} \mu_{j}(k)\left\{P_{j}(k-1)+\left[\hat{X}_{j}(k-1)-\right.\right. \\
& \left.\hat{X}(k-1)]\left[\hat{X}_{j}(k-1)-\hat{X}(k-1)\right]^{T}\right\}
\end{aligned}
$$

\section{Simulation and result analysis}

Two models $m_{1} 、 m_{1}$ are selected to form the model set $M=\left\{m_{1}, m_{2}\right\}$, the number of the models can be adjusted due to the situation. The noise of model $m_{2}$ is less than model $m_{1}$, that is $\sigma_{Q 1}=100 \sigma_{Q 2}, \sigma_{R 1}=100 \sigma_{R 2}$.

For this simulation, the gyroscope first-order Markov random drift is $0.01\left(^{\circ}\right) / h$, constant drift is $0.1\left(^{\circ}\right) / \mathrm{h}$. The first-order Markov random drift of accelerometer is $0.0001 \mathrm{~g}$. The measurement noise of GNSS receiver is white noise with standard deviation of $15 \mathrm{~m}$. the simulation time is $500 \mathrm{~s}$, the position and orientation error figures of SINS,GNSS/SINS integrated system and the IMM-SINS/GNSS fusion system are as follows:

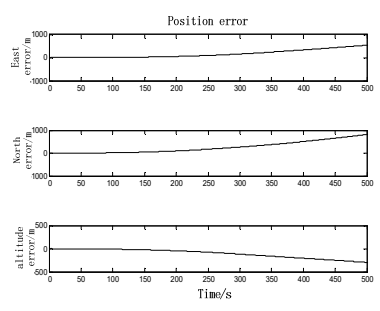

Fig. 2 Position error curve of SINS

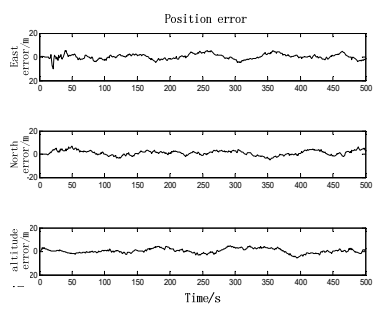

Fig.4 Position error curve of IMM-SINS/GNSS Fig.5 Estimate of SINS attitude misaligned angle

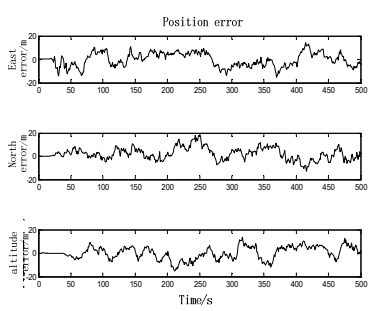

Fig.3 Position error curve of SINS/GNSS

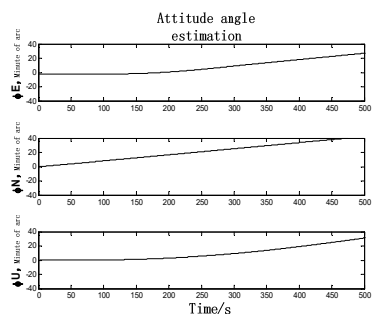




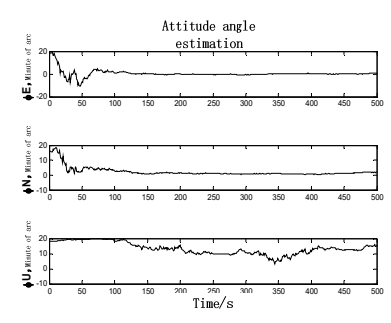

Fig.6

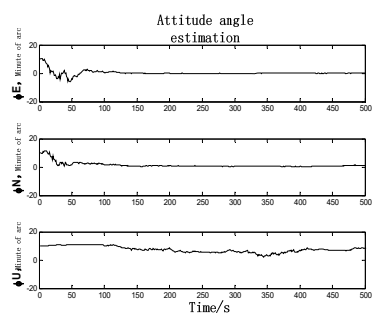

Fig.7

Fig.6 Estimate of SINS/GNSS attitude misaligned angle

Fig.7 Estimate of IMM-SINS/GNSS attitude misaligned angle

Through the analysis of simulation figures, it can be known that: the errors of gyroscope accumulate with time in SINS and can't meet the requirements of aerial photographic system; the error of SINS/GNSS integrated navigation system change sharply while the carrier makes high motivation and the computing of exterior orientation elements may be affected. But the optimized algorithm in this text takes the advantages of IMM and SINS/GNSS, so the result is the best.

\section{Summary}

High precision position and orientation information is very significant for efficiency and productivity in aerial photographic system. This fusion algorithm can realize self-adaptive characteristic in severe weather, high motivation and strong jamming circumstance. Simulation result shows that the precision of this algorithm can meet the requirements of aerial photographic system. In future, the number of models can be changed according to the system parameter and it's motivation characteristics. The algorithm possessed values of engineering application.

\section{Reference}

[1] Petovello M.G. Performance Analysis of an Ultra-Tightly Integrated GPS and Reduce IMU system, ION GNSS 2007, 1-8, USA, 2007.

[2] ZHANG Tong, ZHANG Jun. Study and Comparison of Filtering Methods for Integrated Navigation System[J]. Computer simulation,2009,26(8):33-36.

[3] ZHANG Ke,LIU Haipeng,LI Hengnian,QIAN Shan. SINS/GPS/CNS integrated navigation federal filtering algorithm[J].Joural of Chinese Inertial Technology,2013,21(2):226-230.

[4] XIONG Jian,WEI Linsheng,GUO Hang. SINS/CNS nonlinear tightly integrated method based on additive quaternion[J]. Journal of Chinese Inertial Technology,2012,20(5):596-600.

[5] ZANG Rongchun,CUI Pingyuan,CUI Hutao,JIN Yi.Integrated navigation algorithm based on IMM-UKF[J].Control theory\&Applications,2007,24(4):634-638.

[6] LI X R, BAR-SHALOM Y. Model-Set adaptation in multiple-model estimation for hybrid system[C]//Proc of American Control Conference. San Francisco,CA:[s.n.],1992:1794-1779.

[7] LIANG Yan,CHENG Yongmei. Analysis on the performance and properties of interacting multiple models algorithm[J].control theory\&Applications,2001,18(4):487-492.

[8] GOU Na,WANG Rui.GUO Xiangke,FENG Xiaolin. Design of fuzzy adaptive Kalman Filter in integrated navigation system[J]. Journal of air force engineering university(Natural science edition),2007,8(2):36-39.

[9] YU Feng,LIU Jianye,XIONG Zhi,LIU Dan. Multi-information fusion method of micro satellite attitude determination system[J]. Journal of Shanghai Jiaotong University,2008,42(5):831-835.

[10]QIAN Shan. Research on relative measurement and attitude control for on-orbit servicing spacecraft[D]. Changsha: National University of Defense Technology,2010.

[11]LI Qiao,LIU Jianye,ZHENG Guandong, et al. Novel celestial navigation for satellite[J]. Transactions of Nanjing University of Aeronautics\&Astronautics,2008,25(2):101-105. 
[12]LIU Ruihua, LIU Jianye. A new method of information fusion method in INS/GPS/TRCOM system[J]. Journal of Chinese Inertial Technology, 2001,9(2):29-33. 